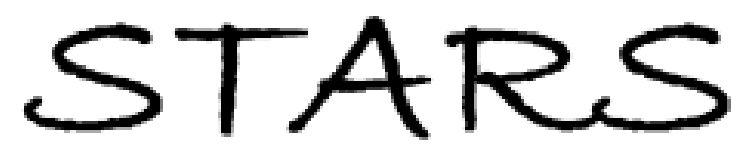

University of Central Florida

STARS

$1-1-2006$

\title{
Strehl ratio and scintillation theory for uplink Gaussian-beam waves: beam wander effects
}

\author{
Larry C. Andrews \\ University of Central Florida \\ Ronald L. Phillips \\ University of Central Florida \\ Richard J. Sasiela \\ Ronald R. Parenti
}

Find similar works at: https://stars.library.ucf.edu/facultybib2000

University of Central Florida Libraries http://library.ucf.edu

This Article is brought to you for free and open access by the Faculty Bibliography at STARS. It has been accepted for inclusion in Faculty Bibliography 2000s by an authorized administrator of STARS. For more information, please contactSTARS@ucf.edu.

\section{Recommended Citation}

Andrews, Larry C.; Phillips, Ronald L.; Sasiela, Richard J.; and Parenti, Ronald R., "Strehl ratio and scintillation theory for uplink Gaussian-beam waves: beam wander effects" (2006). Faculty Bibliography 2000s. 5906.

https://stars.library.ucf.edu/facultybib2000/5906

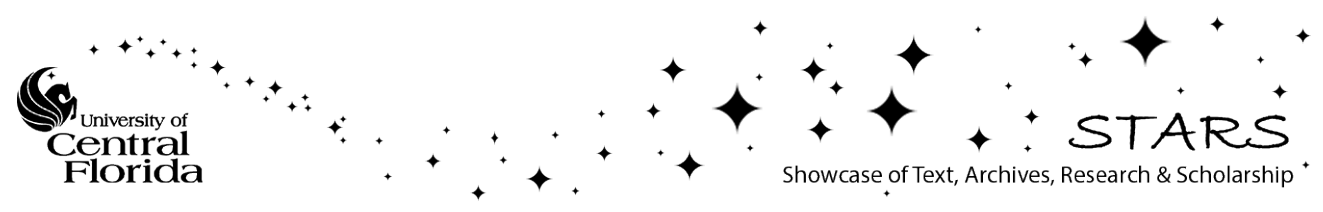




\section{Strehl ratio and scintillation theory for uplink Gaussian-beam waves: beam wander effects}

\author{
Larry C. Andrews, MEMBER SPIE \\ University of Central Florida \\ Department of Mathematics \\ and Florida Space Institute \\ Orlando, Florida 32816
}

\author{
Ronald L. Phillips \\ University of Central Florida \\ Department of Electrical and Computer \\ Engineering and Florida Space Institute \\ Orlando, Florida 32816
}

Richard J. Sasiela
Ronald R. Parenti
Massachusetts Institute of Technology
Lincoln Laboratory
Lexington, Massachusetts 02173-9108

\begin{abstract}
First-order weak-fluctuation Rytov theory predicts that the longitudinal (on-axis) component of the scintillation index of an uplink collimated beam will become significantly smaller as the size of the transmitter aperture increases up to around $100 \mathrm{~cm}$. However, the results of recent computer simulations are at odds with this behavior, and we believe that this discrepancy is due to the fact that the conventional Rytov theory does not correctly account for the effects of beam wander on the scintillation index. We present a theoretical structure that accurately describes far-field irradiance fluctuations caused by uncorrected beam wander. This new theory is validated by demonstrating excellent agreement between the predicted scintillation index and computer code results for both tracked and untracked beams. For many applications of practical interest, such as free-space optical communications, a good understanding of the time-average Strehl ratio is also essential simulation results for this parameter are presented and shown to be in good agreement with the theory. ( ) 2006 Society of Photo-Optical Instrumentation Engineers. [DOI: 10.1117/1.2219470]

Subject terms: scintillation; Rytov theory; beam wander; Strehl ratio.
\end{abstract}

Paper 050656R received Aug. 15, 2005; revised manuscript received Nov. 22 2005; accepted for publication Dec. 7, 2005; published online Jul. 21, 2006.

\section{Introduction}

Over the years, the scientific community has devoted considerable effort to the development of analytic models for predicting irradiance fluctuations associated with a lowestorder Gaussian-beam wave. ${ }^{1-12}$ Irradiance fluctuations, commonly called scintillation, are generally considered to be a fundamental limiting factor in the performance of freespace optical (FSO) communication links and laser radar systems, among other applications. However, in spite of the progress that has been made in developing these scintillation models, there are still some aspects of beam-wave propagation that are not well understood, particularly in regards to convergent beams and uplink collimated beams. For example, first-order weak-fluctuation Rytov theory predicts that the longitudinal component (or on-axis scintillation) of a focused beam along a horizontal path continually decreases as the size of the laser-transmitter aperture increases. ${ }^{2-6}$ Similar on-axis behavior has been predicted for a collimated beam on an uplink path through weak optical turbulence to a satellite. ${ }^{3,7-10}$ In particular, the implied uplink scintillation index deduced from the conventional Rytov theory leads to greatly reduced values of scintillation (orders of magnitude reduction) on the boresight or optical axis of a ground-to-space collimated beam, in which the beam diameter is on the order of 20 to $100 \mathrm{~cm}$. At the same time, the conventional Rytov theory predicts that the radial component of scintillation for a focused beam or an uplink collimated beam increases without bound as the transmitter aperture increases. Because such behavior for any beam is

0091-3286/2006/\$22.00 @ 2006 SPIE physically unrealistic, we reach the conclusion that the conventional Rytov theory is incomplete with regards to these particular cases.

In an early experimental study, Kerr and Dunphy ${ }^{13}$ concluded that the predicted on-axis scintillation reduction for a beam focused on a near-field receiver may not be realized in the presence of significant beam wander. Early theoretical studies addressing power reduction caused by beam wander include those of Esposito, ${ }^{14}$ Fried, ${ }^{15}$ and Titterton. ${ }^{16}$ These particular studies were concerned primarily with developing a probability density function (pdf) model to describe the statistics of transmitter platform pointing jitter in the absence of atmospheric turbulence, similar to the effect of beam wander in turbulence. More recently, Kiasaleh ${ }^{17}$ and Steinvall ${ }^{18}$ examined the impact of platform pointing jitter combined with beam scintillation, employing several commonly used pdf models for beam scintillation. Concern with the first-order Rytov theory for focused beams has also been expressed by others such as Banakh and Smalikho. ${ }^{19}$ Although Banakh and Smalikho provided a curve fit to the on-axis scintillation of an uplink beam, none of the previous studies produced a consistent, tractable model of onaxis and off-axis scintillation that combines beam wander effects with the Rytov scintillation theory.

In a recent uplink numerical wave optics simulation study, Dios et al. ${ }^{20}$ showed that the on-axis log-amplitude variance of a collimated beam from ground to space can be much greater than that predicted by the conventional Rytov theory when beam wander is present. Dios et al. argue that beam-wander-induced scintillation must be added to the Rytov approximation in such cases. Baker and Benson ${ }^{21}$ also showed similar discrepancies in the on-axis scintillation between that predicted by the conventional Rytov 
theory and their numerical wave optics simulation results in an uplink path to an altitude of $300 \mathrm{~km}$. Again, the conclusion reached by Baker and Benson is that a large reduction in the on-axis scintillation index of an intermediate-size collimated beam as predicted by the conventional Rytov theory cannot be achieved in the presence of beam wander. By adding tracking wander to the wave optics simulation, the on-axis simulation results and Rytov theory were in better agreement. However, the radial (or off-axis) irradiance fluctuations predicted by the numerical simulation results with tracking wander included ${ }^{21}$ were generally below the results predicted by the conventional Rytov theory.

In this work, we use weak and strong irradiance fluctuation theory to present a further analysis of the behavior of a collimated beam along an uplink path to space, taking into account whether the movement of the short-term wandering beam is being tracked or not. In particular, we develop new expressions for the longitudinal and radial scintillation index components of an uplink propagating beam that are modifications of models based on the conventional Rytov theory. To do this, we develop a new expression for the beam wander displacement associated with an uplink beam that manifests itself as an apparent or "effective pointing error" that must be accounted for in the measured value of the scintillation index when the beam is not perfectly tracked.

\section{Review of the Rytov Theory}

In the context of the conventional Rytov theory, most early investigations into the statistical characteristics of an optical wave propagating through weak atmospheric turbulence were concerned with the log-amplitude variance $\sigma_{\chi}^{2}$ of an infinite plane wave or spherical wave, both of which are limiting cases of the more general lowest-order Gaussianbeam wave. The governing integrals for beam-wave propagation were formulated first by Schmeltzer, ${ }^{1}$ who used the Rytov method. Fried and Seidman ${ }^{2}$ solved these integrals for the longitudinal component of the log-amplitude variance using a Kolmogorov power-law spectrum model for refractive-index fluctuations. Their analysis discussed the transition of a collimated beam from spherical wave to plane-wave propagation for increasing transmitter aperture size. They also predicted a substantial reduction in scintillation for a large-aperture transmitted beam focused on a receiver. Ishimaru ${ }^{4}$ used spectral analysis techniques to obtain expressions for several statistical quantities, including the log-amplitude variance. His general results include points at some radial distance from the boresight and, for a focused beam, predict the same scintillation reduction for the longitudinal component as that of Fried and Seidman. More recent studies of scintillation have also led to the same results. $5,6,8,22$

\subsection{Beam Parameters}

Let us first consider the free-space analysis of a unitamplitude Gaussian-beam wave that in the plane of the transmitter at $z=0$ is described by
$U_{0}(\mathbf{r}, L)=\exp \left(-\frac{r^{2}}{W_{0}^{2}}-i \frac{k r^{2}}{2 F_{0}}\right)$

where $r$ is a vector in the transverse direction, $W_{0}$ is the $1 / e$ field radius of the beam, and $F_{0}$ is the phase front radius of curvature. Also, $k=2 \pi / \lambda$ is the optical wave number and $\lambda$ is wavelength. We use the convention that $F_{0}>0$ for a convergent beam and $F_{0}<0$ for a divergent beam. For a propagation path of length $L$ along the positive $z$ axis, it is convenient to describe free-space propagation by use of two sets of nondimensional beam parameters ${ }^{10}$

$\Theta_{0}=1-\frac{L}{F_{0}}, \quad \Lambda_{0}=\frac{2 L}{k W_{0}^{2}}$,

$\Theta=1+\frac{L}{F}=\frac{\Theta_{0}}{\Theta_{0}^{2}+\Lambda_{0}^{2}}, \quad \Lambda=\frac{2 L}{k W^{2}}=\frac{\Lambda_{0}}{\Theta_{0}^{2}+\Lambda_{0}^{2}}$.

The first set of beam parameters in Eq. (2) characterizes the beam at the transmitter, and the second set in Eq. (3) characterizes the beam at the receiver, where $W$ and $F$ represent the spot size radius and phase front radius of curvature of the beam as viewed in the receiver plane. In some expressions used later, it is also useful to introduce the notations $\bar{\Theta}_{0}=1-\Theta_{0}$ and $\bar{\Theta}=1-\Theta$.

\subsection{Mean Irradiance and Strehl Ratio}

Under the conventional Rytov approximation, the on-axis mean irradiance is ${ }^{10}$

$\langle I(0, L)\rangle=\frac{W_{0}^{2}}{W_{\mathrm{LT}}^{2}}=\frac{W_{0}^{2}}{W^{2}(1+T)}$,

where $W_{\mathrm{LT}}$ is the long-term spot radius under weak irradiance fluctuations, \langle\rangle denotes an ensemble average, and

$$
\begin{aligned}
T= & 4 \pi^{2} k^{2} \int_{0}^{L} \int_{0}^{\infty} \kappa \Phi_{n}(\kappa, z) \\
& \times\left[1-\exp \left(-\frac{\Lambda L \kappa^{2}(1-z / L)^{2}}{k}\right)\right] d \kappa d z,
\end{aligned}
$$

where $\Phi_{n}(\kappa, z)$ is the atmospheric spatial power spectrum. From Eq. (4) we obtain the corresponding weak fluctuation Strehl ratio

$\langle S\rangle=\frac{1}{1+T}$.

\subsection{Scintillation Index}

Under the conventional Rytov theory, the scintillation index of a Gaussian-beam wave is related to the log-amplitude variance by $4,7,10$ 


$$
\begin{aligned}
\sigma_{I}^{2}(r, L)= & 4 \sigma_{\chi}^{2}(r, L) \\
= & 8 \pi^{2} k^{2} L \int_{0}^{1} \int_{0}^{\infty} \kappa \Phi_{n}(\kappa) \exp \left(-\frac{\Lambda L \kappa^{2} \xi^{2}}{k}\right) \\
& \times\left\{I_{0}(2 \Lambda r \kappa \xi)-\cos \left[\frac{L \kappa^{2}}{k} \xi(1-\bar{\Theta} \xi)\right]\right\} d \kappa d \xi
\end{aligned}
$$

where $I_{0}(x)$ is a modified Bessel function of the first kind and $\xi=1-z / L$ is a normalized distance variable. The distinguishing feature of this expression for the scintillation index compared with that for an infinite plane wave or spherical wave is the dependency of Eq. (7) on the radial position in the beam. Because of this dependency, it is sometimes useful to separate the scintillation index into the sum of a radial and a longitudinal component in the form $\sigma_{I}^{2}(r, L)=\sigma_{I, r}^{2}(r, L)+\sigma_{I, l}^{2}(L)$, where we simply rearrange the integrals in Eq. (7) to identify

$$
\begin{aligned}
\sigma_{I, r}^{2}(r, L)= & 8 \pi^{2} k^{2} L \int_{0}^{1} \int_{0}^{\infty} \kappa \Phi_{n}(\kappa) \exp \left(-\frac{\Lambda L \kappa^{2} \xi^{2}}{k}\right) \\
& \times\left[I_{0}(2 \Lambda r \kappa \xi)-1\right] d \kappa d \xi \\
\sigma_{I, l}^{2}(L)= & 8 \pi^{2} k^{2} L \int_{0}^{1} \int_{0}^{\infty} \kappa \Phi_{n}(\kappa) \exp \left(-\frac{\Lambda L \kappa^{2} \xi^{2}}{k}\right) \\
& \times\left\{1-\cos \left[\frac{L \kappa^{2}}{k} \xi(1-\bar{\Theta} \xi)\right]\right\} d \kappa d \xi .
\end{aligned}
$$

If we use the Kolmogorov spectrum

$$
\Phi_{n}(\kappa, z)=0.033 C_{n}^{2}(z) \kappa^{-11 / 3}
$$

where we assume $C_{n}^{2}(z)$ varies as a function of propagation distance $z$, Eqs. (8) and (9) can be reduced, respectively, to

$$
\sigma_{I, r}^{2}(r, L)=14.50 k^{7 / 6} L^{5 / 6} \Lambda^{5 / 6} \frac{r^{2}}{W^{2}} \int_{0}^{L} C_{n}^{2}(z)(1-z / L)^{5 / 3} d z
$$

$$
\begin{aligned}
\sigma_{I, l}^{2}(L)= & 8.70 k^{7 / 6} L^{5 / 6} \operatorname{Re} \int_{0}^{L} C_{n}^{2}(z)\left\{i^{5 / 6}(1-z / L)^{5 / 6}\right. \\
& \left.\times[1-(\bar{\Theta}+i \Lambda)(1-z / L)]^{5 / 6}-\Lambda^{5 / 6}(1-z / L)^{5 / 3}\right\} d z,
\end{aligned}
$$

where $i=\sqrt{-1}$ and Re denotes the real part. Next, we introduce suitable modifications of these standard scintillation models to account for the effects of beam wander.

\section{Beam Wander Model}

A finite optical beam will randomly wander (snake-like) as it propagates through the atmosphere. Over short time periods the beam profile at the receiver can become highly skewed from a Gaussian profile and, in addition, the entire spot may move off the boresight (see Fig. 1). We refer to the instantaneous point of maximum irradiance as the "hot spot" [Fig. 1(a)]. This combined phenomenon, which is a refinement of what is commonly called beam wander, can (a)

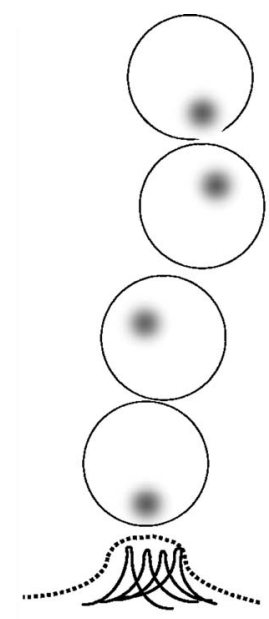

(b)

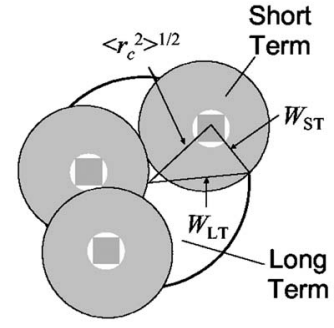

Fig. 1 (a) Beam wander as described by movement of the "hot spot" (instantaneous center) within the beam. (b) The long-term spot size is the result of beam wander, beam breathing, and diffraction. The shaded circles depict random motion of the short-term beam in the receiver plane.

be characterized statistically by the variance of the magnitude of the wander displacement. It has been shown experimentally that beam wander has a time constant on the order of the beam diameter over the transverse wind speed, and thus its effect can be mitigated with the use of a fasttracking transmitter. ${ }^{23}$

In addition to references already given, beam wander has been extensively studied by a number of other researchers. $^{24-30}$ It is known that beam wander is caused mostly by large-scale turbulence, and for that reason the analysis is often based on the geometrical optics method (GOM) where diffraction effects are neglected. Chernov ${ }^{25}$ and Beckmann ${ }^{26}$ both used the GOM in their analyses, but did not consider the finite size of the beam. $\mathrm{Chiba}^{27}$ included the finite size of the beam in his analysis for the case of a collimated beam, and Churnside and Lataitis ${ }^{23}$ used the GOM to develop an expression for the variance of the beam wander displacement that included both collimated and focused beams. By use of the Markov approximation, a more comprehensive analysis of beam wander that includes the effects of diffraction has also been developed. ${ }^{28,29}$ In this latter approach, the analysis for the displacement variance led to the introduction of a spatial filter that suppresses the influence of turbulent cell sizes smaller than the beam. Fante $^{30}$ developed a relation between the long-term beam spot size, the short-term beam spot size, and the variance of displacement of the short-term beam using fourth-order statistics. Tavis and Yura ${ }^{31}$ developed a short-term beam profile based entirely on the small-scale effect of turbulence.

\subsection{General Model}

Beam wander at the receiver plane can be modeled as if it arises from a random tilt angle at the transmitter plane, similar to angle-of-arrival fluctuations of a reciprocal propagating wave with the receiver diameter replaced by the transmitter beam diameter. The combined movement of the hot spot and short-term beam centroid as depicted by the illustrations in Fig. 1 paints out the large outer circle 
over a long time period that we call the long-term spot size $W_{\mathrm{LT}}$. Based on this model, we write the long-term spot size in Eq. (4) as an averaged statistical measure that can be written in the form

$$
W_{\mathrm{LT}}^{2}=\underbrace{W^{2}}_{\text {diffrac. }}+\underbrace{W^{2} T_{\mathrm{SS}}}_{\substack{\text { sm-scale } \\
\text { spread }}}+\underbrace{W^{2} T_{\mathrm{LS}}}_{\begin{array}{c}
\text { large-scale } \\
\text { beam wander }
\end{array}}=W_{\mathrm{ST}}^{2}+<r_{c}^{2}\rangle,
$$

where we have partitioned the quantity $T$ from Eq. (5) into a sum of small-scale (SS) contributions $T_{\mathrm{SS}}$ and large-scale (LS) contributions $T_{\mathrm{LS}}$. The first term in the middle expression in Eq. (13) is that due to free-space diffraction spreading, the center term of this expression is additional spreading caused by turbulent scale sizes smaller than the beam, and the last term describes the beam wander displacement variance of the beam in the receiver plane caused by turbulent scale sizes larger than the beam. The first two terms together in the middle expression define the short-term beam radius $W_{\mathrm{ST}}$. It must be noted that as a result of using $W$ to define the $1 / e^{2}$ radius of the irradiance profile, our beam wander term $W^{2} T_{\mathrm{LS}}=\left\langle r_{c}^{2}\right\rangle$ in Eq. (13) is a factor of 2 larger than the parameter that other investigators ${ }^{20,21,23,30}$ have used to characterize beam centroid wander.

To emphasize the effect of large-scale turbulence on beam wander, we model the last term on the right in Eq. (13) under weak irradiance fluctuations by the expression

$$
\begin{aligned}
\left\langle r_{c}^{2}\right\rangle= & W^{2} T_{\mathrm{LS}}=4 \pi^{2} k^{2} W^{2} \int_{0}^{L} \int_{0}^{\infty} \kappa \Phi_{n}(\kappa, z) H_{\mathrm{LS}}(\kappa, z) \\
& \times\left\{1-\exp \left[-\frac{\Lambda L \kappa^{2}(1-z / L)^{2}}{k}\right]\right\} d \kappa d z,
\end{aligned}
$$

where we have introduced the large-scale filter function ${ }^{28,29}$

$$
\begin{aligned}
H_{\mathrm{LS}}(\kappa, z)= & \exp \left[-\kappa^{2} W^{2}(z)\right]=\exp \left\{-\kappa^{2} W_{0}^{2}\left[\left(1-z / F_{0}\right)^{2}\right.\right. \\
& \left.\left.+\left(2 z / k W_{0}^{2}\right)^{2}\right]\right\}
\end{aligned}
$$

The Gaussian filter function in Eq. (15) only permits random inhomogeneities equal to the beam size and larger to contribute to beam wander, thereby eliminating small-scale effects that lead to the short-term spot size in Eq. (13). In Eq. (15), $W(z)$ is the free-space beam radius at variable distance $z(0<z<L)$ from the transmitter.

It can be shown that the last term in the argument of the exponential function in Eq. (15) can be neglected in our analysis, because it describes free-space diffraction effects. That is, we use a geometrical optics approximation to evaluate the integral in Eq. (14), and, owing to the filter function in Eq. (14) that eliminates the effects of large spatial wave numbers (i.e., small-scale turbulence), we can further invoke the approximation

$$
1-\exp \left[-\frac{\Lambda L \kappa^{2}(1-z / L)^{2}}{k}\right] \approx \frac{\Lambda L \kappa^{2}(1-z / L)^{2}}{k}
$$

$\frac{\Lambda L \kappa^{2}(1-z / L)^{2}}{k} \ll 1$

Consequently, with these simplifications we find that Eq. (14) becomes

$$
\left\langle r_{c}^{2}\right\rangle=7.25 L^{2} W_{0}^{-1 / 3} \int_{0}^{L} C_{n}^{2}(z)(1-z / L)^{2}\left|1-z / F_{0}\right|^{-1 / 3} d z
$$

We note that the scaling constant in Eq. (17) is roughly 3.5 times the constant in Eq. (10) of Churnside and Lataitis ${ }^{23}$ for a single-axis displacement variance when rewritten in terms of $W_{0}$.

\section{Irradiance Statistics for an Uplink Path}

For applications involving propagation along a slant path near the ground, it is standard practice to assume the structure parameter $C_{n}^{2}(z)$ is a function of propagation distance $z$. Moreover, in propagation along an uplink path to space, it is customary to use a $C_{n}^{2}(h)$ profile model to describe variations in the structure parameter as a function of altitude $h$. Several $C_{n}^{2}(h)$ profile models, including both day and night models, are used by the technical community for groundto-space or space-to-ground applications. ${ }^{32}$ One of the most widely used models is the Hufnagel-Valley $(\mathrm{H}-\mathrm{V})$ model described by

$$
\begin{aligned}
C_{n}^{2}(h)= & 0.00594\left(\frac{V}{27}\right)^{2}\left(10^{-5} h\right)^{10} \exp \left(-\frac{h}{1000}\right)+2.7 \\
& \times 10^{-16} \exp \left(-\frac{h}{1500}\right)+A \exp \left(-\frac{h}{100}\right),
\end{aligned}
$$

where $V$ is a pseudo-wind and $A$ is a parameter commonly set to $A=1.7 \times 10^{-14} \mathrm{~m}^{-2 / 3}$. By also selecting $V=21 \mathrm{~m} / \mathrm{s}$, the model is then referred to as the $\mathrm{HV}_{5 / 7}$ model or $\mathrm{HV}-21$ model. Although there also exist some models for outer scale as a function of altitude, such as that based on scintillation and ranging (SCIDAR) observations at the European Southern Observatory in Chile, ${ }^{33}$ such models are still generally viewed as controversial by many in the community. Moreover, the authors are not aware of any acceptable inner scale models as a function of altitude. As a result, for the geometry associated with an uplink path, it is customary to assume a Kolmogorov spectrum of the form in Eq. (8), where the effects from inner scale and outer scale are not taken into account.

It was shown many years ago that the principle of reciprocity applies to the performance of a telescope as measured by its effective coherence size in atmospheric turbulence, whether it is functioning as part of a transmitter or as part of a receiver. ${ }^{34}$ Thus, by invoking the principle of reciprocity, we argue that beam wander at the receiver plane can be modeled as if it arises from a random tilt at the transmitter plane. In this sense, the random tilt is similar to angle-of-arrival fluctuations of a reciprocal propagating wave with the receiver diameter replaced by the transmitter beam diameter.

In the case of a collimated beam, Eq. (17) reduces to 


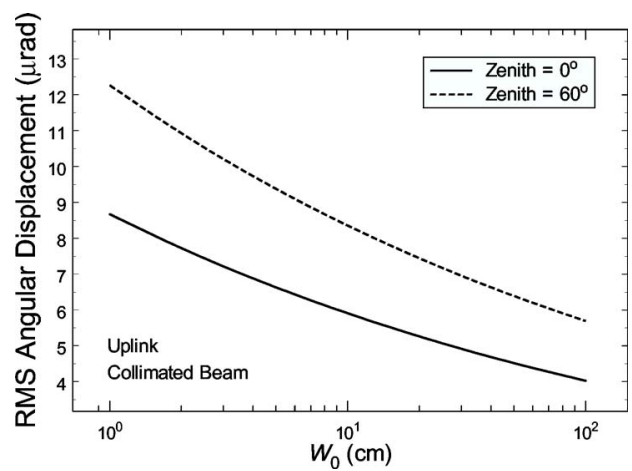

Fig. 2 The rms angular beam wander in microradians plotted as a function of beam radius at the receiver for a transmitter on the ground and a satellite in space.

$\left\langle r_{c}^{2}\right\rangle=7.25 L^{2} W_{0}^{-1 / 3} \int_{0}^{L} C_{n}^{2}(z)(1-z / L)^{2} d z$.

Adapted specifically for an uplink path at zenith angle $\zeta$, we can rewrite the beam wander displacement variance in Eq. (19) in the form

$$
\begin{aligned}
\left\langle r_{c}^{2}\right\rangle= & 7.25\left(H-h_{0}\right)^{2} \sec ^{3}(\zeta) W_{0}^{-1 / 3} \\
& \times \int_{h_{0}}^{H} C_{n}^{2}(h)\left(1-\frac{h-h_{0}}{H-h_{0}}\right)^{2} d h \\
\approx & 0.54\left(H-h_{0}\right)^{2} \sec ^{2}(\zeta)\left(\frac{\lambda}{2 W_{0}}\right)^{2}\left(\frac{2 W_{0}}{r_{0}}\right)^{5 / 3},
\end{aligned}
$$

where $H$ is the altitude of the receiver and $h_{0}$ is the altitude of the transmitter (which we generally assume is zero). In arriving at our result, we have used the approximation

$\int_{h_{0}}^{H} C_{n}^{2}(h)\left(1-\frac{h-h_{0}}{H-h_{0}}\right)^{2} d h \approx \int_{h_{0}}^{H} C_{n}^{2}(h) d h=\mu_{0}$,

in the second line of Eq. (20) and introduced the atmospheric coherence diameter $r_{0}$ (i.e., Fried's parameter) defined by

$r_{0}=\left(0.423 \mu_{0} k^{2} \sec \zeta\right)^{-3 / 5}$.

Here, we see that the beam wander variance involves the free-space diffraction angle $\lambda / 2 W_{0}$ and the tilt phase fluctuations averaged over the transmitter aperture, the latter of which are on the order of $\left(2 W_{0} / r_{0}\right)^{5 / 3}$. Except for the scaling constant, Eq. (20) is the same result as Eq. (40) in Fante $^{30}$ and Eq. (16) in Baker and Benson. ${ }^{21}$

In Fig. 2 we illustrate the effect of the root-mean-square (rms) angular beam wander $\sqrt{\left\langle r_{c}^{2}\right\rangle} / L$ in microradians for various size collimated beams from ground to space at zenith angles $\zeta=0$ and $60 \mathrm{deg}$. Note that the angular displacement is greatest for smaller beams and steadily decreases with increasing beam radius.

\subsection{Strehl Ratio}

Within the laser beam-control community, the standard measure of system performance is the Strehl ratio, which is the ratio of the measured on-axis irradiance and the diffraction-limited irradiance. Most of the published theories relating to Strehl estimates assume a uniformly illuminated aperture of diameter $D$, and if turbulence-induced phase distortions are close to the plane of the transmitter, it has been shown that the Maréchal approximation is reasonably accurate for weak-turbulence conditions

$\langle S\rangle \approx \exp \left[-\left(D / r_{0}\right)^{5 / 3}\right]$.

Improved estimates have been derived by Andrews and Phillips. ${ }^{10}$ Under weak fluctuation conditions, they show [see also Eq. (6)]

$\langle S\rangle=\left[1+\left(D / r_{0}\right)^{5 / 3}\right]^{-1}, \quad D / r_{0} \ll 1$,

whereas under a broad range of phase distortions their result is

$\langle S\rangle=\left[1+\left(D / r_{0}\right)^{5 / 3}\right]^{-6 / 5}, \quad 0 \leqslant D / r_{0}<\infty$.

Both Eqs. (24) and (25) can be rewritten for a Gaussian beam of radius $W_{0}$ using the reciprocity property. ${ }^{34}$ The relationship $D=2^{3 / 2} W_{0}$ has also been incorporated, which derives from an optimal match between the far-field irradiance profiles associated with a circular aperture and an infinite Gaussian. Thus, the far-field Strehl ratio deduced from Eq. (25) for uncorrected turbulence effects can be written as

$\langle S\rangle_{\text {uncorrected }}=\left[1+2^{5 / 2}\left(W_{0} / r_{0}\right)^{5 / 3}\right]^{-6 / 5}$.

Most applications of optical beam propagation involve some form of active target tracking, so Strehl and scintillation estimates for tilt correction are of great practical importance. Closed-loop beam tilt control is essential for any device application that is highly sensitive to far-field irradiance fluctuations. Free-space optical communication systems are generally power limited and severely impacted by turbulence-induced fading, so jitter mitigation is often a primary consideration in the design of the beam-control hardware. Because many communication links are bidirectional, beam-pointing information is usually derived from the apparent direction of the incoming light from the companion transceiver. Tracking errors tend to be dominated by servo-loop bandwidth limitations, and point-ahead anisoplanatism may be important if the platform velocity is high. However, an assessment of performance with imperfect beam control is outside the scope of this work.

Fried $^{35}$ was one of the first investigators to examine the behavior of a tracked beam, and his results show that the normalized irradiance is significantly larger than that for an untracked beam when the $D / r_{0}$ ratio is close to unity. An analysis of the simulation results provided by Lincoln Laboratory shows that the tilt-corrected Strehl is closely approximated by the following expression

$$
\begin{aligned}
\langle S\rangle_{\text {till corrected }} \approx & \left\{1+\left[5.56-\frac{4.84}{1+0.04\left(W_{0} / r_{0}\right)^{5 / 3}}\right]\right. \\
& \left.\times\left(W_{0} / r_{0}\right)^{5 / 3}\right\}^{-6 / 5} .
\end{aligned}
$$

We note here that Eqs. (25) and (27) are consistent with 
Noll's observation $^{36}$ that the uncorrected phase variance at the transmitter plane is $1.03\left(D / r_{0}\right)^{5 / 3}$ and the magnitude of the tilt component is $0.896\left(D / r_{0}\right)^{5 / 3}$. Because the tilt component leads to an expression for the Strehl that is valid only when $D / r_{0}<1$, Eq. (27) was developed as an accurate modification of the tilt-corrected Strehl for both small and large values of the ratio $D / r_{0}$. The numerical constants that appear in Eq. (27) were chosen on the basis of a parametric optimization process applied to both uplink and horizontalpath simulation data.

\subsection{Wander-Induced Pointing Error Variance}

As defined before, beam wander describes movement of the short-term beam centroid, but also includes movement of the hot spot around the beam centroid that can produce a skewed short-term Gaussian-beam profile. The combined effects of short-term beam centroid and hot spot movements are caused by turbulent atmospheric scale sizes on the order of the beam and larger. However, long-term beam-wander-induced scintillation is caused by a subset of the large scale sizes that are bounded above by the atmospheric coherence width $r_{0}$. This leads to an effective "pointing error displacement" $\sigma_{\mathrm{pe}}$ of the beam that is distinct from the centroid displacement, and results in an increase of the detected on-axis scintillation index over that deduced from the conventional Rytov theory.

More specifically, for beams satisfying $W_{0}<r_{0}$, the detected scintillation at the receiver due to a wander-induced pointing displacement error is the result of turbulent cells near the transmitter on the order of the beam size and larger, up to the atmospheric coherence width $r_{0}$. Hence, to determine the variance of the wander-induced pointing error, we now introduce a modified version of the spatial frequency filter function in Eq. (15) that eliminates the effects of scale sizes that are larger than the atmospheric coherence width. This filter function is described by

$$
\begin{aligned}
H(\kappa, \xi)_{\text {point error }}= & \exp \left[-\kappa^{2} W_{0}^{2}\left(\Theta_{0}-\bar{\Theta}_{0} \xi\right)^{2}\right] \\
& \times\left[1-\exp \left(-\frac{\kappa^{2}}{\kappa_{r}^{2}}\right)\right]
\end{aligned}
$$

where $\xi=1-z / L$ is a normalized distance variable and $\kappa_{r}$ is the spatial frequency cutoff associated with the atmospheric coherence width, i.e.,

$\kappa_{r}=\frac{C_{r}}{r_{0}}$.

The parameter $C_{r}$ is a scaling constant that is typically in the range of 1 to $2 \pi$, which depends on the definition of $r_{0}$ (e.g., along horizontal paths the atmospheric coherence width may be associated with a spherical wave). When $W_{0}>r_{0}$, the same filter function in Eq. (28) is used, but in this case the amplitude of the filter tends to zero as the ratio $W_{0} / r_{0}$ increases; consequently, we expect the wanderinduced pointing error to diminish.

To calculate the pointing error variance $\sigma_{\mathrm{pe}}^{2}$ in the case of a collimated beam, we substitute the filter function in Eq. (28) into the integral in Eq. (5), multiplied by $W^{2}$, to find

$$
\begin{aligned}
\sigma_{\mathrm{pe}}^{2}= & 4 \pi^{2} L k^{2} W^{2} \int_{0}^{1} \int_{0}^{\infty} \kappa \Phi_{n}(\kappa, \xi) H(\kappa, \xi)_{\mathrm{jitter}} \\
& \times\left[1-\exp \left(-\frac{\Lambda L \kappa^{2} \xi^{2}}{k}\right)\right] d \kappa d \xi \\
= & 0.54\left(H-h_{0}\right)^{2} \sec ^{2}(\zeta)\left(\frac{\lambda}{2 W_{0}}\right)^{2}\left(\frac{2 W_{0}}{r_{0}}\right)^{5 / 3} \\
& \times\left[1-\left(\frac{C_{r}^{2} W_{0}^{2} / r_{0}^{2}}{1+C_{r}^{2} W_{0}^{2} / r_{0}^{2}}\right)^{1 / 6}\right] \\
= & \left\langle r_{c}^{2}\right\rangle\left[1-\left(\frac{C_{r}^{2} W_{0}^{2} / r_{0}^{2}}{1+C_{r}^{2} W_{0}^{2} / r_{0}^{2}}\right)^{1 / 6}\right],
\end{aligned}
$$

where we have employed the geometrical optics approximation in Eq. (16) once again. From Eq. (30) we see that the pointing error $\sigma_{\mathrm{pe}}$ is proportional to rms beam wander displacement $\sqrt{\left\langle r_{c}^{2}\right\rangle}$. Also, depending on the value of the ratio $2 W_{0} / r_{0}$, we can easily deduce from Eq. (30) the following asymptotic behavior:

$\sigma_{\mathrm{pe}}^{2} \sim\left\{\begin{array}{l}\left(\frac{\lambda}{2 W_{0}}\right)^{2}\left(\frac{2 W_{0}}{r_{0}}\right)^{5 / 3}\left(H-h_{0}\right)^{2} \sec ^{2}(\zeta), \quad 2 W_{0} / r_{0} \ll 1 \\ \left(\frac{\lambda}{2 W_{0}}\right)^{2}\left(\frac{r_{0}}{2 W_{0}}\right)^{1 / 3}\left(H-h_{0}\right)^{2} \sec ^{2}(\zeta), \quad 2 W_{0} / r_{0} \gg 1\end{array}\right.$

In particular, when $2 W_{0} \ll r_{0}$, we recognize that $\sigma_{\mathrm{pe}}^{2} \approx\left\langle r_{c}^{2}\right\rangle$. And although the beam wander variance can be quite large in this case, the ratio $\sigma_{\mathrm{pe}}^{2} / W^{2}$ will be small because of the large beam radius $W$ in the receiver plane [e.g., see Eq. (32)]. In the other asymptotic case $2 W_{0} \gg r_{0}$, the pointing error variance $\sigma_{\mathrm{pe}}^{2}$ will tend to zero. That is, when $2 W_{0}$ $\gg r_{0}$, the short-term beam no longer wanders in the same sense but breaks up into smaller pieces due to the loss of spatial coherence across the beam. As a consequence, we expect beam wander to have little influence on the on-axis scintillation index in either asymptotic regime in Eq. (31).

\section{Uplink Scintillation Index}

Beam wander effects are contained in the on-axis behavior of the irradiance through the long-term spot size $W_{\mathrm{LT}}$ defined by Eq. (4), but this is not the case for the on-axis scintillation index. Thus, for an untracked beam, the longitudinal component of the detected scintillation will necessarily increase over that predicted by the Rytov theory due to the impact of the random wandering of the short-term beam spot on and off boresight.

\subsection{Untracked Beam}

In view of the previous comments, we need to redefine the components of the detected scintillation index, depending on whether the beam is tracked or not. Because $\sigma_{\mathrm{pe}}$ represents an effective pointing error displacement of a Gaussian beam at the receiver, we define the longitudinal component for an untracked beam by the expression 


$$
\begin{aligned}
\sigma_{I, l}^{2}(L)_{\text {untracked }}= & 5.95\left(H-h_{0}\right)^{2} \sec ^{2}(\zeta)\left(\frac{2 W_{0}}{r_{0}}\right)^{5 / 3}\left(\frac{\alpha_{\mathrm{pe}}}{W}\right)^{2} \\
& +8.70 \mu_{u 1} k^{7 / 6}\left(H-h_{0}\right)^{5 / 6} \sec ^{11 / 6}(\zeta) .
\end{aligned}
$$

In arriving at this result, we have introduced the notation

$\mu_{u 1}=\operatorname{Re} \int_{h_{0}}^{H} C_{n}^{2}(h)\left\{\xi^{5 / 6}[\Lambda \xi+i(1-\bar{\Theta} \xi)]^{5 / 6}-\Lambda^{5 / 6} \xi^{5 / 3}\right\} d h$,

and incorporated the relation $\left(L>>k W_{0}^{2}\right)$

$$
\begin{aligned}
& 14.53 \Lambda^{5 / 6} k^{7 / 6}\left(H-h_{0}\right)^{5 / 6} \sec ^{11 / 6}(\mathrm{~s}) \\
& \quad \times \int_{h_{0}}^{H} C_{n}^{2}(h)\left(1-\frac{h-h_{0}}{H-h_{0}}\right)^{5 / 3} d h \approx 5.95\left(\frac{2 W_{0}}{r_{0}}\right)^{5 / 3} .
\end{aligned}
$$

In addition, we set $\alpha_{\mathrm{pe}}=\sigma_{\mathrm{pe}} / L$.

The first term on the right-hand side of Eq. (32) is simply the conventional radial term in Eq. (11) for the scintillation index evaluated at the displacement $r=\sigma_{\mathrm{pe}}$. We note that this on-axis scintillation model is consistent with that described by Dios et al. ${ }^{20}$ concerning beam-wander-induced scintillation. At radial distances $r>\sigma_{\mathrm{pe}}$, we define the detected scintillation index throughout the beam profile by

$$
\begin{aligned}
\sigma_{I}^{2}(r, L)_{\text {untracked }}= & 5.95\left(H-h_{0}\right)^{2} \sec ^{2}(\zeta) \\
& \times\left(\frac{2 W_{0}}{r_{0}}\right)^{5 / 3}\left(\frac{\alpha_{r}-\alpha_{\mathrm{pe}}}{W}\right)^{2} U\left(\alpha_{r}-\alpha_{\mathrm{pe}}\right) \\
& +\sigma_{I, l}^{2}(L)_{\text {untracked }}, \quad \alpha_{r}<W / L,
\end{aligned}
$$

where $\alpha_{r}=r / L$ and $U(x)$ is the unit step function

$$
U(x)=\left\{\begin{array}{ll}
1, & x>0 \\
0, & x<0
\end{array} .\right.
$$

Thus, in this model it is assumed that the detected scintillation index in Eq. (35) is equal to the longitudinal component throughout the circular (pointing error) domain $0 \leqslant r$ $\leqslant \sigma_{\mathrm{pe}}$

\subsection{Tracked Beam-Part 1}

The notion of "tracking a beam" can vary from system to system, depending on a chosen technique. In particular, it might mean tracking the hot spot in the beam or removing tilt from the wandering beam as discussed in Sec. 4.1, among others. In practice, the technique of hot-spot tracking is challenging when the fluctuations become strong, because the beam tends to break up into multiple beams, thereby creating more than one hot spot. For the theoretical model developed in this section, we define a tracked beam as one based on the removal of rms beam wander effects. In this case, the longitudinal component is described by

$\sigma_{I, l}^{2}(L)_{\text {tracked }} \equiv \sigma_{B}^{2}=8.70 \mu_{u 1} k^{7 / 6}\left(H-h_{0}\right)^{5 / 6} \sec ^{11 / 6}(\zeta)$.

We recognize this expression as that based on the conventional Rytov theory. ${ }^{10}$ By removing the rms beam wander displacement from the radial component, the scintillation index at off-axis points throughout the beam profile becomes

$$
\begin{aligned}
\sigma_{I}^{2}(r, L)_{\text {tracked }}= & 8.70 \mu_{u 1} k^{7 / 6}\left(H-h_{0}\right)^{5 / 6} \sec ^{11 / 6}(\zeta) \\
& +5.95\left(H-h_{0}\right)^{2} \sec ^{2}(\zeta)\left(\frac{2 W_{0}}{r_{0}}\right)^{5 / 3} \\
& \times\left(\frac{\alpha_{r}-\sqrt{\left\langle\alpha_{c}^{2}\right\rangle}}{W}\right)^{2} U\left(\alpha_{r}-\sqrt{\left\langle\alpha_{c}^{2}\right\rangle}\right) \\
& 0 \leqslant \alpha_{r}<W / L
\end{aligned}
$$

where $\sqrt{\left\langle\alpha_{c}^{2}\right\rangle}=\sqrt{\left\langle r_{c}^{2}\right\rangle} / L$. Note that the final result in Eq. (38) is not the same as that derived by the conventional Rytov theory. In particular, Eq. (38) predicts somewhat lower offaxis scintillation values than that from the conventional Rytov theory.

\subsection{Tracked Beam-Part 2}

The tilt-corrected numerical simulations developed at Lincoln Laboratory and used in this study impose "perfect tracking" in the sense that sensor noise and bandwidth limitations are neglected. The Lincoln Laboratory code infers the beam position from the instantaneous far-field beam profile and measures the irradiance centroid. Results published by other researchers may be based on different tracking schemes.

Here we develop a model for the tracked beam case to compare with simulation results derived from the Lincoln Laboratory code. To do so, we remove the rms "tilt" from the far-field beam, using the two-axis Zernike tilt variance defined by ${ }^{7}$

$T_{Z}^{2}=0.32\left(\frac{\lambda}{2 W_{0}}\right)^{2}\left(\frac{2 W_{0}}{r_{0}}\right)^{5 / 3}$.

By removing the rms Zernike tilt displacement from the rms beam wander displacement, we obtain the tilt-corrected pointing error variance, given by

$\sigma_{\mathrm{pe}, \mathrm{TC}}^{2}=\left(\sqrt{\left\langle r_{c}^{2}\right\rangle}-L T_{z}\right)^{2}\left[1-\left(\frac{C_{r}^{2} W_{0}^{2} / r_{0}^{2}}{1+C_{r}^{2} W_{0}^{2} / r_{0}^{2}}\right)^{1 / 6}\right]$.

In terms of Eq. (40), the longitudinal component for a tiltcorrected beam is given by the expression

$$
\begin{aligned}
\sigma_{I, l}^{2}(L)_{\text {tracked }}= & 5.95\left(H-h_{0}\right)^{2} \sec ^{2}(\zeta)\left(\frac{2 W_{0}}{r_{0}}\right)^{5 / 3}\left(\frac{\alpha_{\mathrm{pe}, \mathrm{TC}}}{W}\right)^{2} \\
& +8.70 \mu_{u 1} k^{7 / 6}\left(H-h_{0}\right)^{5 / 6} \sec ^{11 / 6}(\zeta)
\end{aligned}
$$

where $\alpha_{\mathrm{pe}, \mathrm{TC}}=\sigma_{\mathrm{pe}, \mathrm{TC}} / L$. Note that Eq. (41) has the same form as Eq. (32) for an untracked beam, but with the tiltcorrected wander-induced pointing angle $\alpha_{\mathrm{pe}, \mathrm{TC}}$ taking the place of $\alpha_{\text {pe }}$. That is, the tilt-corrected beam still has a residual pointing error term in the detected scintillation index.

\subsection{Strong Irradiance Fluctuations}

Under strong irradiance fluctuations such as those caused by large-diameter beams $\left(W_{0}>r_{0}\right)$ and/or large zenith angles, the weak fluctuation results presented before are not 
adequate. Building off the uplink scintillation model for an uplink spherical wave under strong irradiance fluctuations, ${ }^{12}$ the corresponding scintillation model for the longitudinal component of an uplink, collimated beam with removal of the rms beam wander effects is given by

$$
\begin{aligned}
\sigma_{I, l}^{2}(L)_{\text {tracked }}= & \exp \left\{\frac{0.49 \sigma_{B}^{2}}{\left[1+0.56(1+\Theta) \sigma_{B}^{12 / 5}\right]^{7 / 6}}\right. \\
& \left.+\frac{0.51 \sigma_{B}^{2}}{\left(1+0.69 \sigma_{B}^{12 / 5}\right)^{5 / 6}}\right\}-1,
\end{aligned}
$$

where $\sigma_{B}^{2}$ is the longitudinal component defined by Eq. (37). Under these conditions, Eq. (42) is valid for all beam diameters and zenith angles. The scintillation index throughout the beam profile with the removal of rms beam wander effects becomes

$$
\begin{aligned}
\sigma_{I}^{2}(r, L)_{\text {tracked }}= & 5.95\left(H-h_{0}\right)^{2} \sec ^{2}(\zeta)\left(\frac{2 W_{0}}{r_{0}}\right)^{5 / 3} \\
& \times\left(\frac{\alpha_{r}-\sqrt{\left\langle\alpha_{c}^{2}\right\rangle}}{W}\right)^{2} U\left(\alpha_{r}-\sqrt{\left\langle\alpha_{c}^{2}\right\rangle}\right) \\
& +\sigma_{I, l}^{2}(L)_{\text {tracked }},
\end{aligned}
$$

where the longitudinal component is defined by Eq. (42). In the case of an untracked beam, the extension of Eq. (32) takes the form

$$
\begin{aligned}
\sigma_{I, l}^{2}(L)_{\text {untracked }}= & 5.95\left(H-h_{0}\right)^{2} \sec ^{2}(\zeta)\left(\frac{2 W_{0}}{r_{0}}\right)^{5 / 3}\left(\frac{\alpha_{\mathrm{pe}}}{W}\right)^{2} \\
& +\exp \left\{\frac{0.49 \sigma_{B}^{2}}{\left[1+0.56(1+\Theta) \sigma_{B}^{12 / 5}\right]^{7 / 6}}\right. \\
& \left.+\frac{0.51 \sigma_{B}^{2}}{\left(1+0.69 \sigma_{B}^{12 / 5}\right)}\right\}-1 .
\end{aligned}
$$

Similarly, the general expression for the untracked beam at any radial position within the profile of the free-space beam is

$$
\begin{aligned}
\sigma_{I}^{2}(r, L)_{\text {untracked }}= & 5.95\left(H-h_{0}\right)^{2} \sec ^{2}(\zeta) \\
& \times\left(\frac{2 W_{0}}{r_{0}}\right)^{5 / 3}\left(\frac{\alpha_{r}-\alpha_{\mathrm{pe}}}{W}\right)^{2} U\left(\alpha_{r}-\alpha_{\mathrm{pe}}\right) \\
& +\sigma_{I, l}^{2}(L)_{\text {untracked }}, \quad \alpha_{r}<W / L,
\end{aligned}
$$

where the longitudinal component is defined by Eq. (44). In the case of a tracked beam using tilt-correction methods, we simply replace $\alpha_{\mathrm{pe}}$ in Eqs. (44) and (45) with $\alpha_{\mathrm{pe}, T C}$.

The validity of models in Eqs. (43)-(45) generally restricts the wander-induced pointing error scintillation term [e.g., the first term on the right in Eqs. (43)-(45)] to values on the order of unity or less. Thus, at low Earth orbit (LEO), we find that Eqs. (43)-(45) may be valid for all beam sizes at the transmitter within zenith angles $\zeta$ $\leqslant 60 \mathrm{deg}$, but at geostationary orbit (GEO), this limitation may restrict their validity to beams for which $W_{0} / r_{0} \leqslant 1$, even at zero zenith. However, because the removal of beam wander effects or tilt correction tends to reduce scintilla-

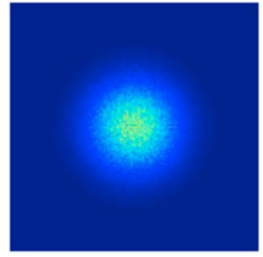

$W_{0} / r_{0} \ll 1$

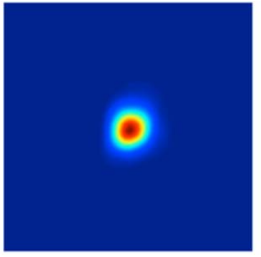

$W_{0} / r_{0} \approx 1$

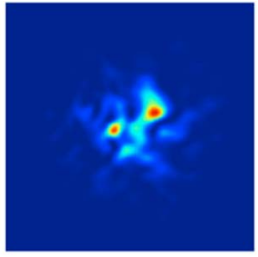

$\mathrm{W}_{0} / \mathrm{r}_{0} \gg 1$
Fig. 3 The beam profile within the receiver plane undergoes a series of dramatic changes as the $W_{0} / r_{0}$ ratio increases. When this parameter is small, the beam is nearly diffraction limited and the irradiance fluctuations are log normal with unity mean. Tilt effects dominate when the $W_{0} / r_{0}$ is close to one, and as this ratio becomes large, beam breakup occurs and saturation is achieved. Distinct differences are found in the statistical behavior of the irradiance in each of these three regimes.

tion, the validity of the resulting tracked scintillation models may extend to beams larger than those in the untracked case.

\section{Numerical Wave Optics Simulation Study}

The primary validation tool for the analytical expressions developed in this investigation is the wave propagation code, POPS, developed at Lincoln Laboratory. This 3-D time-dependent program derives from MOLLY, which was written at Lincoln in the late 1980's to study turbulence and thermal blooming effects. Both programs incorporate subroutines to generate random Kolmogorov phase screens, ${ }^{37}$ and provide a means to propagate from one screen to the next using a 2-D Fresnel transformation. ${ }^{38}$ As the beam propagates from screen to screen, the Talanov scale transformation $^{39}$ is imposed to maintain a constant ratio between the beam size and the dimensions of the field discretization grid. An arbitrary number of phase screens can be created to simulate the desired $C_{n}^{2}$ profile, and each screen can be moved perpendicular to the beam in accordance with the wind velocity profile for time-dependent simulations. Statistical behavior is typically characterized by running 100,000 independent realizations of the propagation channel. POPS is a state of the art C-based code with a web-browser interface, which currently functions on a 16-node Xeon processor cluster.

For statistical investigations, the output of the POPS code is generally configured to record the irradiance at a small number of grid points within the receiver plane. To obtain a better understanding of the physical processes that drive beam scintillation, it is often useful to study selected images of the entire profile. As illustrated in Fig. 3, one can clearly distinguish three regimes of behavior based on the $W_{0} / r_{0}$ ratio. Log-normal statistics are only observed when $W_{0} / r_{0} \ll 1$, where the conventional Rytov approximation is applicable. Tilt effects dominate for untracked beams when $W_{0} / r_{0} \sim 1$, and saturation due to beam breakup occurs when $W_{0} / r_{0} \gg 1$. In the latter case, the statistics can be characterized as a negative exponential function. Figure 3 demonstrates that beam-wave scintillation is not a simple process, but rather the result of a complex set of phenomena that are strongly dependent on the propagation geometry and the turbulence profile. 


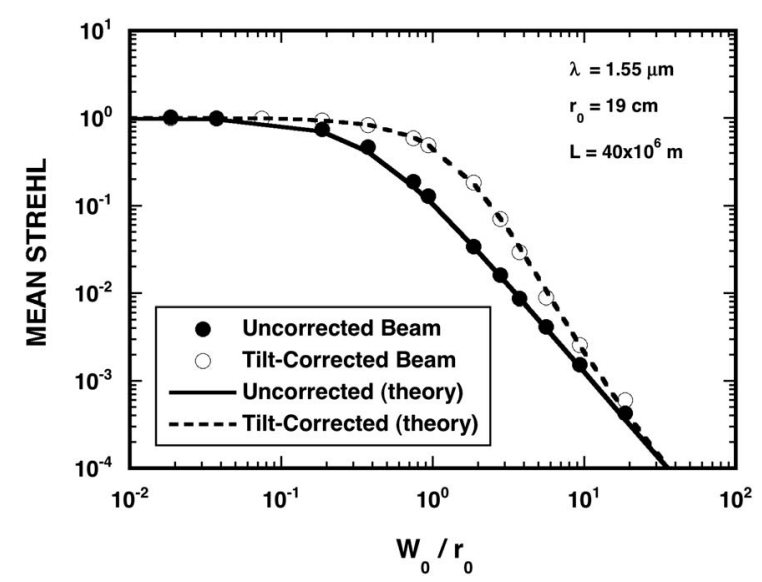

Fig. 4 Comparison of theory and simulation results for uncorrected and tilt-corrected mean Strehl ratio. The solid circles (uncorrected) and solid triangles (tilt-corrected) represent simulation results, and the dashed curves are theoretical results.

Lastly, it must be noted that in all of our investigations the target is placed at the geometrical focus of the beam, so that it is always situated in the Fourier transform plane of the transmitter. When this constraint is applied, the observed scintillation effects are insensitive to the value of the uplink propagation range as long as the target is well outside of the atmosphere. Our use of the term "collimated beam" in the included illustrations is in the context of ground-to-space propagation geometries, where the receiver range is large compared to the Rayleigh range. For these scenarios, there is no practical distinction between collimated and focused beams.

\section{Comparison of Theory with Simulation Results}

Comparisons of uplink-path simulation output and mean Strehl predictions based on Eqs. (26) and (27) are shown in Fig. 4 as a function of $W_{0} / r_{0}$. Simulation results are based on the $\mathrm{HV}_{5 / 7}$ model using a Kolmogorov spectrum. In this simulation, a $1.55-\mu \mathrm{m}$ Gaussian beam is propagated vertically to a satellite in geostationary orbit at a range of $40,000 \mathrm{~km}$. The atmospheric coherence width in this case is $r_{0}=19 \mathrm{~cm}$.

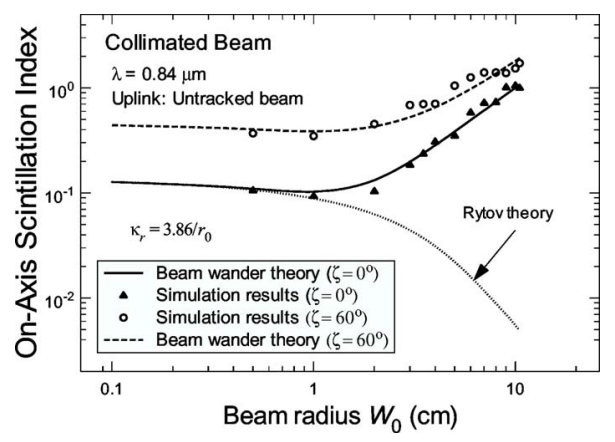

Fig. 5 The longitudinal component of scintillation as a function of beam radius. The filled triangles and open circles represent simulation results for an untracked beam (courtesy of Dios). Theoretical results for untracked beams are also shown, as is that based on the conventional Rytov theory (dotted curve).

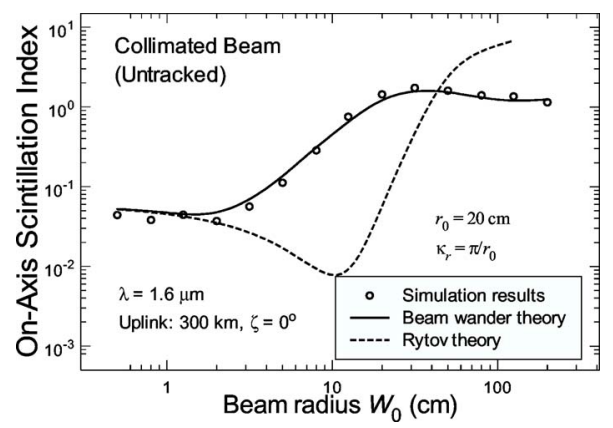

Fig. 6 On-axis scintillation index for an untracked collimated beam as a function of beam radius at the transmitter. The dashed curve represents the conventional Rytov theory. (Simulation results courtesy of Baker.)

In Fig. 5 we show simulation results ${ }^{40}$ as a function of beam radius for the uplink longitudinal component of an untracked collimated beam in geosynchronous orbit (GEO) with zenith angles 0 and $60 \mathrm{deg}$. Simulation results are based on the $\mathrm{HV}_{5 / 7}$ model using a Kolmogorov spectrum and wavelength $\lambda=0.84 \mu \mathrm{m}$. Theoretical curves (solid and dashed lines) obtained from Eq. (32) are also shown for comparison. However, so as to provide a good fit with these particular simulation results, we selected $C_{r}=3.86$ (i.e., $\kappa_{r}$ $\left.=3.86 / r_{0}\right)$. The dotted curve corresponds to Eq. (37) based on the conventional Rytov theory. Here we see close agreement of the beam wander theoretical model with simulation results at both zenith angles.

In Figs. 6 and 7 we show the simulation results of Baker and Benson ${ }^{21}$ for an uplink, untracked, collimated beam with wavelength $\lambda=1.6 \mu \mathrm{m}$ to a target at $300 \mathrm{~km}$, zero zenith angle, and $r_{0}=20 \mathrm{~cm}$. In both figures, the $\mathrm{H}-\mathrm{V}_{5 / 7}$ model was combined with a Kolmogorov spectrum to produce the results. In Fig. 6, we plot the longitudinal component of the scintillation index as a function of beam radius at the transmitter, whereas in Fig. 7 we show the scintillation index as a function of off-axis distance for a collimated beam with $W_{0}=5 \mathrm{~cm}$. Also shown in both figures are theoretical results deduced from Eqs. (44) and (45), respectively. In this case, we found a good fit between theory and simulation results by selecting $\kappa_{r}=\pi / r_{0}$. Because $\sigma_{\mathrm{pe}}$ $\approx 30 \mathrm{~cm}$, the theoretical curve in Fig. 7 does not increase

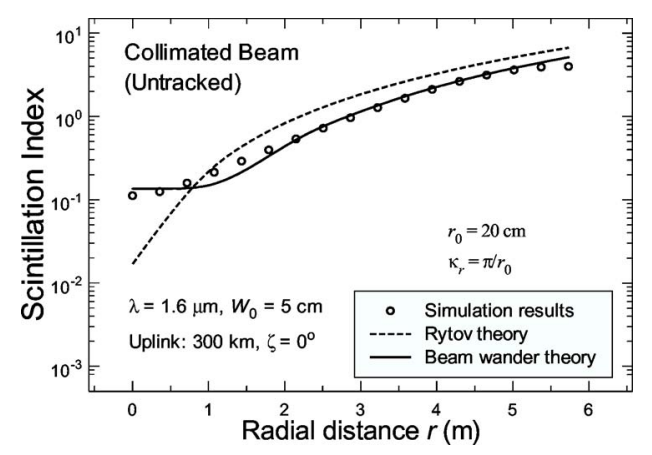

Fig. 7 Scintillation index for an untracked collimated beam of radius $5 \mathrm{~cm}$ as a function of off-axis distance. The dashed curve represents the conventional Rytov theory. (Simulation results courtesy of Baker.) 


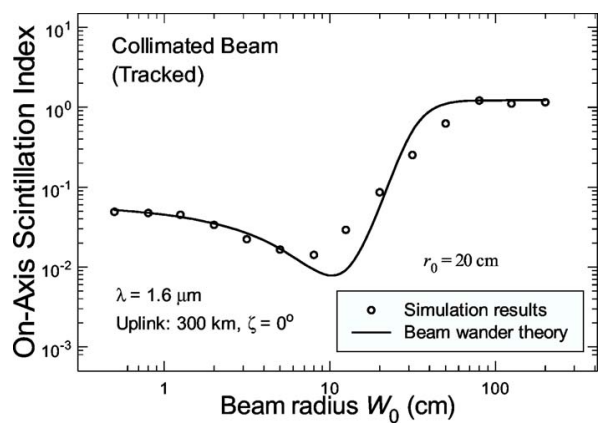

Fig. 8 On-axis scintillation index for a tracked (beam wander removed) collimated beam as a function of beam radius at the transmitter. (Simulation results courtesy of Baker.)

with values of $r$ until $r>\sigma_{\mathrm{pe}}$. For comparison, we also show in both figures the scintillation index predicted by the conventional Rytov theory (dashed curves).

Similar simulation results and theoretical curves are illustrated in Figs. 8 and 9, but this time for the case of a tracked beam. In the simulation results, the "tracking" was accomplished by moving the center of the beam to the boresight in each realization before averaging. Therefore, the simulation procedure should produce somewhat different scintillation values after averaging than those obtained in the theoretical results by first averaging and then removing the rms beam wander displacement, as indicated by Eqs. (42) and (43). In Fig. 8, we plot the on-axis simulation results of Baker and Benson ${ }^{21}$ for a tracked collimated beam as a function of beam radius, and in Fig. 9 we plot the simulation results as a function of off-axis distance for an initial 5 -cm radius beam. Note that because $\sqrt{\left\langle r_{c}^{2}\right\rangle} \approx 2 \mathrm{~m}$, there is no appreciable increase in the simulation results or the theoretical scintillation index until the radial distance exceeds $2 \mathrm{~m}$. The dashed curve is based on the conventional Rytov theory for off-axis fluctuations.

Lastly, in Fig. 10 we plot simulation results generated at Lincoln Laboratory for uncorrected (untracked) and tiltcorrected (tracked) uplink collimated beams to a receiver at zenith and at a range of $40 \times 10^{6} \mathrm{~m}$. Also shown are theoretical results from the beam wander theory given by Eq. (44) with $\alpha_{\mathrm{pe}}$ replaced by $\alpha_{\mathrm{pe}, T C}$ (solid and dotted curves) and the conventional Rytov theory (dashed curve). As in

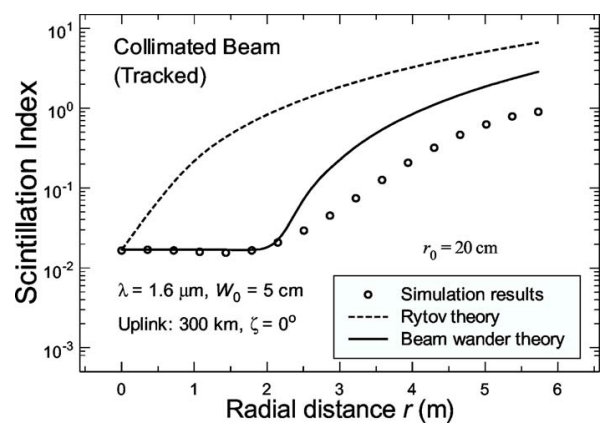

Fig. 9 Scintillation index for a tracked (beam wander removed) collimated beam of radius $5 \mathrm{~cm}$ as a function of off-axis distance. The dashed curve represents the conventional Rytov theory. (Simulation results courtesy of Baker.)

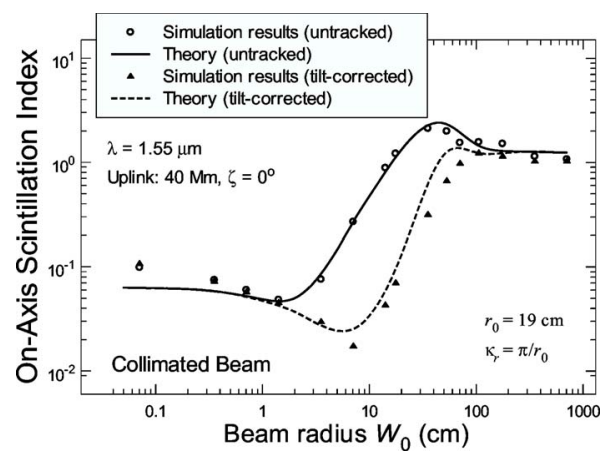

Fig. 10 On-axis scintillation index for untracked and tilt-corrected collimated beams as a function of beam radius at the transmitter. The theoretical curves were calculated for a LEO satellite.

Figs. 6-9, we found a good fit between theory and simulation results with $\kappa_{r}=\pi / r_{0}$. To avoid numerical difficulties associated with the theoretical model for large beams and GEO ranges, the theoretical results shown in Fig. 10 were generated using a 300-km range (LEO) rather than the $40-\mathrm{Mm}$ range of the simulation data. Actual scintillation results at the two ranges should be the same. That is, the untracked simulation results developed at Lincoln Laboratory for vertical propagation to a range of $40 \mathrm{Mm}$ are nearly identical to those generated by Baker and Benson ${ }^{21}$ in Fig. 6 for a target at zenith and a range of $300 \mathrm{~km}$.

\section{Discussion}

It has long been recognized that the conventional Rytov theory for a Gaussian-beam wave is somewhat incomplete with respect to predicting the on-axis scintillation index of a propagating focused beam along a horizontal path or that of an uplink collimated (or focused) beam to space. Moreover, early publications suggested that the reason the Rytov theory was deficient was that it did not include the effects of beam wander, which will generally increase the on-axis scintillation index over that predicted by the Rytov theory. More recent publications based on simulation studies have led to renewed interest in this topic and its impact on freespace optical communication links. In this work, we have developed a theoretical model for the scintillation index of an untracked, uplink collimated beam to space that accounts for the observed increase in scintillation through an induced pointing error caused by beam wander. Theoretical results from these models are compared with our own simulation results as well as those of other researchers.

Unlike previous researchers, we define beam wander as a combination of hot spot movement within the beam as well as that which involves movement of the whole shortterm beam centroid in the receiver plane. We attribute the combined movements to refractive-index scale sizes that are on the order of the beam and larger. The variance $\left\langle r_{c}^{2}\right\rangle$ of beam wander displacement derived here can be related to earlier results by noting $\left\langle r_{c}^{2}\right\rangle=2\left\langle\beta^{2}\right\rangle$, where $\left\langle\beta^{2}\right\rangle$ denotes the beam centroid variance associated with the largest scale sizes. Although both centroid and hot spot movements contribute to the long-term spot size $W_{\mathrm{LT}}$ in the receiver plane, only those scale sizes roughly on the order of the atmospheric coherence width $r_{0}$ can contribute to the wander- 


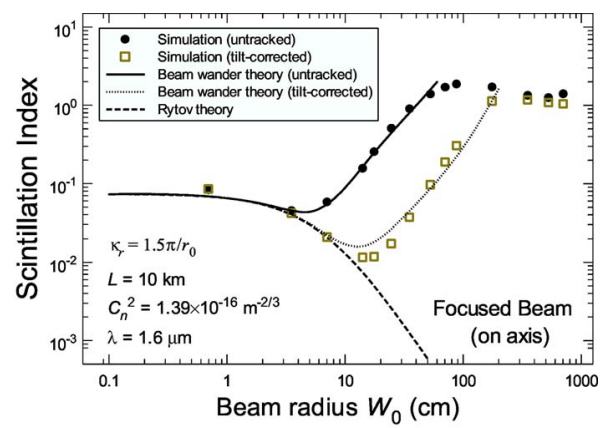

Fig. 11 Simulation results and theoretical curves for the scintillation index of a focused beam on a 10-km horizontal path as a function of beam spot radius at the transmitter.

induced increase in the on-axis scintillation index. As a result, we find the effective wander-induced pointing error $\sigma_{\text {pe }}$ at the receiver to be much smaller than the rms beam wander displacement $\sqrt{\left\langle r_{c}^{2}\right\rangle}$, except in the case of very small beams at the transmitter.

Next, we wish to comment on our choice of scaling constant $C_{r}$ that appears in Eq. (29), namely $\kappa_{r}=C_{r} / r_{0}$. As stated before, we expect this scaling constant to lie within the range of 1 to $2 \pi$, although we have not established its exact value. To compare our theory with simulation results obtained by somewhat different techniques employed by various researchers, we changed the value of the scaling constant by a small amount, depending on the simulation results used for comparison. For example, when comparing our theory with the uplink simulation results of Dios, ${ }^{40}$ we set $C_{r}=3.86$, whereas in comparing our theory with Baker and Benson ${ }^{21}$ and with our own uplink results, we set $C_{r}$ $=\pi$. Hence, the proper choice of scaling constant is still an open question.

Comparisons of the theoretical models for the scintillation index of untracked beams shown in Figs. 6 and 7 show good agreements with the simulation results, both on and off the optical axis of the beam. The agreement between simulation results and the theoretical models for the tracked beam case (based on removing beam wander effects) shown in Figs. 8 and 9 were also reasonably good, but not as good as for the untracked beam case. We attribute the difference in the tracked beam case primarily to a difference in technique between the theoretical model and the simulation results of removing beam wander effects. The theoretical model for the tilt-corrected scintillation index shown in Fig. 10 also provides good agreement with the simulation results. In an earlier study, we made a similar comparison between theoretical models and simulation results for an untracked focused beam on a horizontal path. ${ }^{41}$ We show these simulation results, and more recent simulation results for the tilt-corrected case, along with theoretical curves for both the untracked and tilt-corrected beams in Fig. 11.

Theoretical models for the mean Strehl ratio for an untracked beam and a tilt-corrected beam are also presented here. Comparison of these Strehl ratio models with the simulation results shows excellent agreement for the farfield Strehl ratio in both cases considered.

\section{Acknowledgments}

This work was sponsored by the Department of the Air Force under Air Force contract FA8721-05-C-0002. Opinions, interpretations, conclusions, and other recommendations are those of the authors and are not necessarily endorsed by the United States Government.

\section{References}

1. R. A. Schmeltzer, "Means, variances and covariances for laser beam propagation through a random medium," Q. Appl. Math. 24, 339-354 (1967).

2. D. L. Fried and J. B. Seidman, "Laser beam scintillations in the atmosphere," J. Opt. Soc. Am. 57, 181-185 (1967).

3. D. L. Fried, "Scintillation of a ground-to-space laser illuminator," $J$. Opt. Soc. Am. 57, 980-983 (1967).

4. A. Ishimaru, Wave Propagation and Scattering in Random Media, IEEE Press, Piscataway, New Jersey (1997).

5. W. B. Miller, J. C. Ricklin, and L. C. Andrews, "Log-amplitude variance and wave structure function: a new perspective for Gaussian beams," J. Opt. Soc. Am. A 11, 1653-1660 (1993).

6. W. B. Miller, J. C. Ricklin, and L. C. Andrews, "Effects of the refractive index spectral model on the irradiance variance of a Gaussian beam," J. Opt. Soc. Am. A 11, 2719-2726 (1994).

7. R. J. Sasiela, Electromagnetic Wave Propagation in Turbulence, Springer, New York (1994).

8. J. D. Shelton, "Turbulence-induced scintillation on Gaussian-beam waves: theoretical predictions and observations from a laserilluminated satellite," J. Opt. Soc. Am. A 12, 2172-2181 (1995).

9. L. C. Andrews, R. L. Phillips, and P. T. Yu, "Optical scintillations and fade statistics for a satellite-communication system," Appl. Opt. 34, 7742-7751 (1995); "Optical scintillations and fade statistics for a satellite-communication system: Errata,” Appl. Opt. 36, 6068 (1997).

10. L. C. Andrews and R. L. Phillips, Laser Beam Propagation through Random Media, SPIE Optical Engineering Press, Bellingham, WA, (1998).

11. L. C. Andrews, M. A. Al-Habash, C. Y. Hopen, and R. L. Phillips, "Theory of optical scintillation: Gaussian-beam wave model," Waves Random Media 11, 271-291 (2001).

12. L. C. Andrews, R. L. Phillips, and C. Y. Hopen, Laser Beam Scintillation with Applications, SPIE Press, Bellingham, WA (2001).

13. J. R. Kerr and J. R. Dunphy, "Experimental effects of finite transmitter apertures on scintillations," J. Opt. Soc. Am. 63, 1-8 (1973).

14. R. Esposito, "Power scintillations due to the wandering of the laser beam," Proc. IEEE 55, 1533-1534 (1967).

15. D. L. Fried, "Statistics of laser beam fade induced by pointing jitter," Appl. Opt. 12, 422-423 (1973).

16. P. J. Titterton, "Power reduction and fluctuations caused by narrow laser beam motion in the far field," Appl. Opt. 12, 423-425 (1973).

17. K. Kiasaleh, "On the probability density function of signal intensity in free-space optical communications systems impaired by pointing jitter and turbulence," Opt. Eng. 33(11), 3748-3757 (1994).

18. O. Steinvall, "Performance of laser tracking of small targets during turbulence and beam jitter," Opt. Eng. 43(7), 1609-1621 (2004).

19. V. A. Banakh and I. N. Smalikho, "Statistical characteristics of the laser beam propagating along vertical and sloping paths through a turbulent atmosphere," Proc. SPIE 1968, 303-311 (1993).

20. F. Dios, J. A. Rubio, A. Rodriguez, and A. Camerón, "Scintillation and beam wander analysis in an optical ground station-satellite uplink," Appl. Opt. 43, 3866-3873 (2004).

21. G. J. Baker and R. S. Benson, "Gaussian beam scintillation on ground to space paths: the importance of beam wander," Proc. SPIE $\mathbf{5 5 5 0}$ 225-235 (2004).

22. W. B. Miller, J. C. Ricklin, and L. C. Andrews, "Scintillation of initially convergent Gaussian beams in the vicinity of the geometric focus," Appl. Opt. 34, 7066-7073 (1995).

23. J. H. Churnside and R. J. Lataitis, "Wander of an optical beam in the turbulent atmosphere," Appl. Opt. 29, 926-930 (1990).

24. A. Belmonte, "Feasibility study for the simulation of beam propagation: consideration of coherent lidar performance," Appl. Opt. 39, 5428-5445 (2000)

25. L. A. Chernov, Wave Propagation in a Random Medium, Dover, New York (1967).

26. P. Beckmann, "Signal degeneration in laser beams propagated through a turbulent atmosphere," Radio Sci. 69D, 629-640 (1965).

27. T. Chiba, "Spot dancing of the laser beam propagated through the turbulent atmosphere," Appl. Opt. 10, 2456-2461 (1971).

28. V. I. Klyatskin and A. I. Kon, "On the displacement of spatially bounded light beams in a turbulent medium in the Markovianrandom-process approximation," Radiophys. Quantum Electron. 15, 1056-1061 (1972).

29. V. L. Mironov and V. V. Nosov, "On the theory of spatially limited 
light beam displacements in a randomly inhomogeneous medium," $J$. Opt. Soc. Am. 67, 1073-1080 (1977).

30. R. L. Fante, "Electromagnetic beam propagation in turbulent media," Proc. IEEE 63, 1669-1692 (1975).

31. M. T. Tavis and H. T. Yura, "Short-term average irradiance profile of an optical beam in a turbulent medium," Appl. Opt. 15, 2922-2931 (1976).

32. R. R. Beland, "Propagation through atmospheric optical turbulence," in The Infrared and ElectroOptical Systems Handbook, F. G. Smith, Ed., Vol. 2, Chap. 2, SPIE Optical Engineering Press, Bellingham, WA (1993)

33. C. E. Coulman, J. Vernin, Y. Coqueognoit, and J. L. Caccia, "Outer scale of turbulence appropriate to modeling refractive-index structure profiles," Appl. Opt. 27, 155-160 (1988).

34. D. L. Fried and H. T. Yura, "Telescope-performance reciprocity for propagation in a turbulent medium," J. Opt. Soc. Am. 62, 600-602 (1972).

35. D. L. Fried, "Optical resolution through a randomly inhomogeneous medium for very long and very short exposures," J. Opt. Soc. Am. 56, 1372-1379 (1966)

36. R. J. Noll, "Zernike polynomials and atmospheric turbulence," $J$. Opt. Soc. Am. 66, 207-211 (1976).

37. B. J. Herman and L. A. Strugala, "Method for inclusion of lowfrequency contributions in numerical representation of atmospheric turbulence," Proc. SPIE 1221, 183-192 (1990).

38. J. W. Goodman, Introduction to Fourier Optics, McGraw-Hill, New York (1968).

39. V. I. Talanov, "Focusing of light in cubic media," JETP Lett. 11, 199 (1970).

40. F. Dios, Private Communications.

41. L. C. Andrews, R. L. Phillips, R. J. Sasiela, and R. Parenti, "Beamwander effects on the scintillation index of a focused beam," Proc. SPIE 5793, 28-37 (2005).

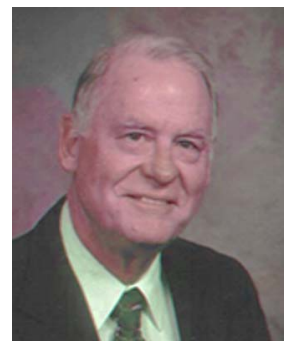

Larry C. Andrews is a professor of mathematics at the University of Central Florida and an associate member of the College of Optics/CREOL. He is also an associate member of the Florida Space Institute. Previously, he held a faculty position at TriState University and was a staff mathematician with the Magnavox Company, antisubmarine warfare operation. He received a doctoral degree in theoretical mechanics in 1970 from Michigan State University. He has been an active researcher in optical wave propagation through random media for more than 25 years and is the author or co-author of ten textbooks on topics of differential equations, boundary value problems, special functions, integral transforms, wave propagation through random media, and mathematical techniques for engineers. Along with wave propagation through random media, his research interests include special functions, random variables, atmospheric turbulence, and signal processing.

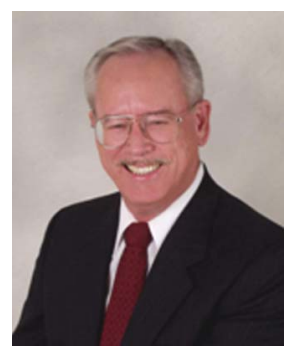

Ronald L. Phillips is director of the Florida Space Institute and a professor in the Department of Electrical and Computer Engineering at the University of Central Florida. $\mathrm{He}$ is also a member of the Department of Mathematics and an associate member of the College of Optics/CREOL. He has held positions on the faculties at Arizona State University and the University of California, San Diego. He received a doctoral degree in electrical engineering in 1970 from Arizona State University. He has been an active researcher in wave propagation through random media for more than 28 years. He was awarded a Senior NATO Postdoctoral Fellowship in 1977 and the American Society for Engineering Education 1983 Medal for outstanding contributions in research. He has co-authored two textbooks on wave propagation through random media and mathematical techniques for engineers. In addition to optical wave propagation, his research interests include optical communications and imaging through atmospheric turbulence.

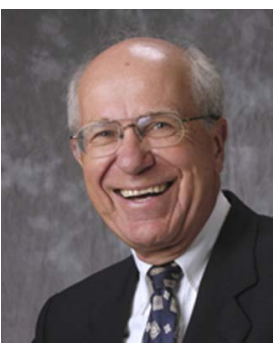

Richard J. Sasiela has been a member of the technical staff at the Massachusetts Institute of Technology, Lincoln Laboratory since 1969. He received his PhD in electrophysics from the Polytechnic Institute of Brooklyn. He has contributed to improving radar systems and measurements, and spent nine years at the Ronald Reagan radar site in the Marshall Islands. His other interests have been in signal processing, image recognition, safety systems for airports, and the propagation of optical and radar beams through the turbulence in the atmosphere. He has written a book on the last topic in which he uses filter functions to reduce problems to integral expressions and Mellin transform to evaluate the integrals. Recently, his research interests have dealt with amplitude statistics of beam propagation and their effect on optical communications systems.

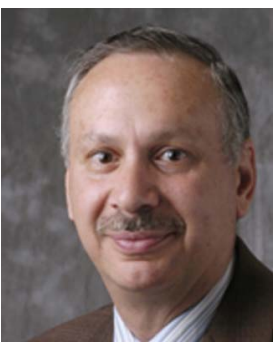

Ronald R. Parenti has been a member of the research staff at the Massachusetts Institute of Technology, Lincoln Laboratory in Lexington Massachusetts since 1974. He received his $\mathrm{PhD}$ in physics from Carnegie Mellon University, where he investigated the use of infrared laser spectroscopy to remotely detect atmospheric pollutants. After joining the Lincoln Laboratory he was initially involved in the development of infrared sensors and associated software for autonomous tactical target detection. Subsequently he became part of a research effort to characterize the performance of high-power laser-beam-control systems, where he developed an expertise in turbulence theory and adaptive optics. His recent work has been primarily devoted to the application of turbulence theory to freespace optical communications. His publications include articles on target discrimination, guide-star adaptive optics for astronomical imaging, and beam-propagation models for free-space communication links. 